\title{
L'évaluation des politiques actives du marché du travail
}

Quels enseignements?

Evaluating active labor market programs: what have we learned?

\section{David Card}

\section{(2) OpenEdition}

\section{Journals}

\section{Édition électronique}

URL : http://journals.openedition.org/travailemploi/6404

DOI : 10.4000/travailemploi.6404

ISSN : 1775-416X

Éditeur

DARES - Ministère du Travail

Édition imprimée

Date de publication : 1 juillet 2014

Pagination : 15-23

ISSN : 0224-4365

\section{Référence électronique}

David Card, «L'évaluation des politiques actives du marché du travail », Travail et Emploi [En ligne], 139 | juillet-septembre 2014, mis en ligne le 01 juillet 2016, consulté le 17 juin 2020. URL : http://

journals.openedition.org/travailemploi/6404; DOI : https://doi.org/10.4000/travailemploi.6404 


\title{
L'évaluation des politiques actives du marché du travail : quels enseignements ?
}

\author{
David Card $\left.{ }^{*}\right)$
}

Cet article propose un panorama sélectif de «ce que nous savons» des succès et des échecs des politiques actives du marché du travail (PAMT). Il commence par brosser une vue d'ensemble de l'histoire de ces politiques, en s'attachant plus particulièrement aux politiques financées par l'État fédéral américain et mises en æuvre aux États-Unis depuis les années 1930. Puis il étudie les différents principes de fonctionnement qui sous-tendent - souvent de manière implicite-la formation professionnelle, les emplois subventionnés ou contrats aidés et les dispositifs d'accompagnement des demandeurs d'emploi proposés, et dresse un bilan de leurs effets. Enfin, il présente une sélection des évaluations marquantes en matière de politiques actives du marché du travail réalisées aux ÉtatsUnis et en France, et indique quelques pistes pour l'élaboration de politiques futures.

Comme l'a rappelé le précédent ministre du Travail, Michel Sapin, en ouverture du colloque sur les politiques actives du marché du travail organisé en février 2013 par la Direction de l'animation de la recherche, des études et des statistiques (Dares) ${ }^{(1)}$, de nombreux États sont actuellement confrontés à des défis importants en termes d'emploi. Outre la France, les États-Unis et bien d'autres pays, frappés de plein fouet par la crise, en subissent encore les conséquences aujourd'hui. Parmi les défis à relever, on compte des taux de chômage toujours plus élevés, et surtout, comme l'a souligné le ministre, celui du chômage des jeunes, un nombre croissant d'entre eux présentant de faibles qualifications et un lien très ténu avec le marché du travail. Dans plusieurs pays vient s'ajouter à cela un troisième problème majeur : les difficultés rencontrées par les travailleurs déclassés. Il s'agit souvent de salariés âgés de 45 à 60 ans environ, qui, alors qu'ils bénéficiaient d'un emploi de bonne qualité avant que ne s'amorce la récession, éprouvent ensuite les plus grandes difficultés à retrouver un poste équivalent à celui qu'ils ont perdu.

Les politiques actives du marché du travail ont été proposées et mises en œuvre dans les contextes les plus variés pour répondre à ce type de problématiques, parmi lesquelles, donc, les problèmes rencontrés par les jeunes actifs, par les travailleurs très peu qualifiés ou encore par les salariés déclassés (LALONDE, 1995). Or, il faut rappeler que sous

(*) University of California Berkeley; card@econ.berkeley.edu (1) Pour en savoir plus sur ce colloque organisé par la Dares à Paris, les 14 et 15 février 2013, voir la page consacrée au colloque sur le site de la Dares : http://travail-emploi.gouv.fr/ etudes-recherches-statistiques-de, 76/etudes-et-recherches,77/ manifestations-et-colloques-de-la, 99/manifestations-etcolloques-passes,688/2013-les-politiques-actives-du, 15797. html; consultée le 27 octobre 2014. l'appellation générale de "politiques actives du marché du travail», on regroupe en réalité toute une série de dispositifs de nature différente, mis en place dans des situations diverses et s'adressant à des types de publics variés. Ainsi, l'une des difficultés que présente la réflexion sur le sujet, notamment si l'on veut faire la synthèse de ce que nous ont appris les dernières décennies en la matière, tient précisément à cette grande hétérogénéité des mesures mises en œuvre et des types de salariés visés. Cependant, pour faire le point sur les connaissances acquises quant à l'efficacité des politiques, on peut, selon nous, s'appuyer sur quatre questions clés.

\section{Quatre questions clés}

Préalablement à toute discussion sur les politiques d'activation, une première question s'impose : à quel grand type de mesures s'intéresse-t-on ? En effet, on a coutume de classer ces politiques en trois grandes catégories, même si, au sein de chacune d'elles, l'hétérogénéité reste entière (voir par exemple KLUve, 2010; CARD et al., 2010). Le premier de ces grands types correspond à ce que nous appellerons les dispositifs de type «aide à la recherche d'emploi». Ces programmes ont commencé à attirer l'attention des chercheurs aux États-Unis au début des années 1990 (GreEnBERG et al., 2005), avant d'être largement diffusés en Europe et ailleurs.

Un deuxième grand type est constitué par les dispositifs de formation. Nous distinguerons ici deux sous-catégories: les programmes de type «scolaire» (classroom programs), consistant à extraire les individus du marché du travail pour les soumettre à un enseignement classique d'une 
durée pouvant atteindre plusieurs années, et les programmes de formation «en emploi» (on the job training programs), où la formation est dispensée dans l'environnement de travail, sur le principe des cursus d'apprentissage, mais pour une durée souvent beaucoup plus brève.

Le troisième grand type de politique active du marché du travail est la création directe d'emplois, les institutions du secteur public étant incitées à embaucher certaines catégories de salariés pour une période donnée. On désignera ces dispositifs sous le nom d' «emplois subventionnés », de «plans de création directe d'emplois » ou encore (dans le contexte français notamment) de «contrats aidés». Dans la plupart des cas, ces mesures ne comportent pas de volet formation à proprement parler; cependant, il arrive que les emplois concernés comprennent une formation sur le terrain.

Une deuxième question clé intervient alors : celle de l'action à court ou long terme des politiques d'activation. Si de nombreux acteurs (et la plupart des évaluations existantes) se concentrent sur une durée relativement courte, la question du bilan à plus long terme paraît au contraire d'une grande importance. En effet, certains projets donnant de bons résultats à court terme ont tendance à voir ceux-ci s'estomper avec le temps, allant dans certains cas jusqu'à porter préjudice aux participants. D'autres au contraire, apparemment peu efficaces dans l'immédiat, voire agissant négativement sur l'emploi des participants à court terme, révèlent un effet bénéfique sur une plus longue durée. Dans la mesure du possible, il convient de privilégier dans l'analyse une perspective à long terme.

Troisième question clé : la mesure fonctionne-telle mieux dans telle ou telle situation particulière, ou pour tel ou tel type de public (LeChNER, Munsch, 2009) ? Existe-t-il des programmes performants en période de tension sur le marché du travail, mais décevants face à un taux élevé de chômage, lorsque les emplois sont rares ? Certains dispositifs fonctionnent-ils pour certains sous-groupes de chômeurs et moins bien pour d'autres ? Aux États-Unis, les candidats défavorisés sont l'objet d'un intérêt non démenti de la part des concepteurs de politiques en faveur de l'emploi. Ainsi, de nombreux projets ont été développés en direction des mères célibataires ayant longtemps vécu des allocations sociales. Les actifs en milieu ou en fin de carrière constituent également une cible privilégiée. Or, ces deux catégories de candidats à l'emploi possèdent des caractéristiques propres, susceptibles d'interagir avec le principe du programme et d'en modifier l'effet.

Enfin, une quatrième et dernière question clé est celle des effets potentiellement indésirables des dispositifs sur les personnes n'en ayant pas bénéficié (voir par exemple Calmfors, 1994). Ainsi, l'une des critiques pouvant être adressées à un plan d'aide à la recherche d'emploi est d'aider une catégorie de personnes (celles qui auront bénéficié du programme) au détriment des autres candidats, sans pour autant créer de nouveaux emplois. Dans ce cas, l'effet positif observé sur les chances des participants est entièrement compensé par l'effet négatif qui en résulte sur les non-participants. Pour aborder cette question, on recourt souvent aux concepts d' «équilibre général» ou d'«effets de déplacement». Si le problème est identifié depuis des années, la recherche en matière d'évaluation commence tout juste à l'étudier directement. Des travaux menés en France nous ont notamment permis de mieux comprendre ce phénomène important.

\section{Les politiques actives, une alternative aux programmes "passifs"}

Pour mieux comprendre les politiques actives du marché de l'emploi, il est utile de les envisager en tant qu'alternatives à des politiques "passives». En effet, dans les pays concernés, la plupart des participants aux programmes actifs sont extraits du groupe des bénéficiaires de politiques passives assurant un revenu. Ainsi, aux États-Unis, la plupart des participants recevaient précédemment l'assurance-chômage (UI, Unemployment Insurance) ou des aides sociales (welfare). En Europe, ceux-ci recevaient soit des allocations-chômage, soit des allocations sociales réservées aux personnes non éligibles à l'assurance-chômage (comme le revenu de solidarité active en France ou l'assistance chômage en Allemagne).

De façon générale, les concepteurs de politiques actives poursuivent simultanément deux grands objectifs. Le premier consiste à inciter les individus à travailler, tout en leur faisant gagner un revenu issu du travail. Le second objectif est de diminuer les dépenses publiques. En règle générale, les concepteurs ne s'intéressent pas directement au bien-être des personnes concernées. Néanmoins, il nous paraît important de soulever cette question. Cherche-t-on vraiment à savoir si participer à une politique active améliore le bien-être des individus, comparativement au fait de bénéficier d'un programme passif ? Or, il s'agit là d'un point décisif, dans la mesure où il pourrait sembler bien plus simple, pour augmenter le taux d'emploi et réduire les dépenses publiques, de supprimer ou diminuer l'aide apportée aux chômeurs par les programmes passifs. De fait, c'est de cette idée que s'inspirent bon nombre de réformes mises en place aux États-Unis au cours des dernières décennies. Or, selon nous, les concepteurs de politiques doivent se fixer un troisième objectif : celui de maintenir constant, ou du moins de ne pas trop diminuer, le bien-être des participants. En ce sens, ajoutons qu'une politique d'activation parvenant à 
accroître le bien-être des participants peut être jugée positive même dans le cas où elle ne diminue que peu, ou pas, les dépenses de l'État comparativement au programme passif auquel elle se substitue. D'un point de vue économique, l'estimation de la plus ou moins grande réussite de la mesure dépendra bien évidemment du poids donné à cette augmentation de la satisfaction des bénéficiaires par rapport à la baisse de celle des contribuables contraints de la financer.

\section{Les principes de fonctionnement des politiques actives du marché du travail}

\section{L'accompagnement à la recherche d'emploi}

Envisageons tout d'abord le cas des dispositifs d'accompagnement à la recherche d'emploi. Il s'agit de programmes conçus à la fois pour intensifier les efforts de recherche des participants et pour améliorer leur efficacité. En règle générale, les personnes inscrites doivent assister à des ateliers leur expliquant où et comment chercher du travail, et se présenter à des rendez-vous réguliers, hebdomadaires ou bimensuels, avec un professionnel chargé de leur suivi. Certains dispositifs offrent aussi des conseils sur la rédaction d'un curriculum vitae (CV), des cours de préparation à l'entretien d'embauche, des réunions de groupe où les membres s'exercent à la pratique de l'entretien et s'encouragent mutuellement à poursuivre leurs recherches.

Ces initiatives appellent deux remarques. La première est qu'elles reposent sur un contrôle des efforts de recherche des participants. Pour les chômeurs inscrits à un tel dispositif, partir en vacances ou travailler au noir tout en continuant à percevoir les allocations devient plus difficile. Par conséquent, c'est là notre seconde remarque, s'engager dans un tel programme peut être jugé moins agréable que le fait de toucher simplement un chèque ou un virement via un système de soutien passif. De fait, un certain nombre de projets expérimentaux ont permis de montrer que la simple menace d'être inscrit à un dispositif d'accompagnement pouvait suffire à accroître le taux d'emploi (voir, par exemple: BLACK et al., 2003). Ainsi, même si le volet «aide à la recherche d'emploi» est en lui-même peu efficace, il peut contribuer à faire entrer les individus dans l'emploi plus rapidement qu'un programme de soutien passif seulement pour éviter le contrôle et les contraintes imposés par le dispositif.

Un troisième aspect propre aux dispositifs d'accompagnement est leur fonction potentielle de «coup de pouce» (nudge) dans le parcours des individus : c'est le principe selon lequel une petite incitation peut entraîner des modifications de comportement importantes. Une abondante recherche ethnographique a montré que les demandeurs d'emploi, et notamment ceux qui le sont depuis longtemps, n'effectuent pas des recherches de façon très active (voir, par exemple : KRUEGER, Mueller, 2011). Il est difficile de dire dans quelle mesure cette recherche «de basse intensité» constitue, ou non, un comportement optimal. Pour certains analystes, le chômage prolongé entraîne un découragement conduisant à l'arrêt des efforts de recherche, alors même que le bénéfice justifiant la recherche est important. Dans un tel contexte, un programme d'accompagnement peut contribuer à lever les barrières psychologiques empêchant les personnes de chercher vraiment un emploi.

Étant donné le contenu de la plupart des programmes d'accompagnement mis en place aux États-Unis, ceux-ci ne sont manifestement pas de nature à avoir des effets à long terme, à moins que l'on ne souscrive à l'idée selon laquelle le chômage de longue durée entraîne des séquelles profondes et durables (scarring effect) sur l'employabilité future, et que l'on considère que pour beaucoup de chômeurs, l'abandon des recherches est plutôt dû à des blocages psychologiques qu'à un choix purement économique en termes de rapport coût bénéfice. Pour que les individus concernés soient véritablement «poussés» à intensifier leurs recherches, il faudrait que ces deux conditions soient remplies. Il est possible que ce soit le cas pour certains sous-groupes, mais pour la plupart des actifs, les effets à long terme d'un dispositif de ce type sont probablement très réduits.

Enfin, un reproche qui peut être formulé à l'encontre de ces dispositifs est leur propension élevée à entraîner des effets de déplacement dans un contexte de récession. Comment expliquer ce phénomène ? Supposons que sur un bassin d'emploi donné, un certain nombre d'emplois soient disponibles: si l'on incite un groupe donné de chômeurs à identifier plus efficacement les postes vacants, sans augmenter dans le même temps la quantité d'offres d'emploi disponibles, on se contente de desservir un autre groupe de demandeurs d'emploi au bénéfice du groupe aidé.

\section{La formation}

Depuis les contributions fondamentales de Jacob Mincer (1958) et Gary BeCKer (1962), les économistes envisagent la formation comme un investissement dans le capital humain. Les cours de type scolaire, d'une part, les formations en situation de travail, d'autre part, constituent dans cette optique deux types d'investissement, donnant lieu à un certain nombre de constatations.

La première n'est qu'à court terme, les personnes en formation, empêchées d'effectuer des tâches productives pour un employeur, gagnent la plupart du temps moins que les personnes ne suivant pas de formation. Cet écart est ce qu'on appelle le «coût 
d'opportunité» de la formation : il sera d'autant plus grand que l'opportunité d'emploi est élevée en l'absence même de programme; à l'inverse, le coût d'opportunité est relativement faible dans le cas où les participants, s'ils n'avaient pas suivi la formation, n'auraient pas non plus travaillé. Ainsi, le coût d'opportunité des formations est plus faible en période de crise, tout comme il est plus faible pour ceux ayant de faibles opportunités d'emploi. La seconde constatation, liée à la première, est qu'une fois leur session de formation achevée, les participants mettent souvent un certain temps pour rattraper ceux qui ne sont pas partis en formation.

Si l'on s'intéresse au plus long terme, les programmes de formation peuvent présenter un bilan positif. Pour mettre en contexte le résultat escompté, notons que la plupart des études portant sur les retombées économiques de la formation supposent que chaque année d'étude de type scolaire accroît les revenus de 10 à $15 \%$ par an (voir par exemple CARD, 1999). À ma connaissance, aucun programme de formation n'exige un plus haut niveau d'investissement qu'une année complète de cours de type scolaire, et nombreux sont les programmes nécessitant un investissement moins intense. On ne doit donc pas s'attendre à les voir augmenter les revenus des participants au-delà des 10 à $15 \%$ par an évoqués plus haut. En pratique, un effet escompté de l'ordre de $5 \%$ paraît sans doute plus raisonnable.

Un troisième constat, tiré des nombreuses recherches portant sur la qualité de l'enseignement, est que la formation dispensée dans le cadre des politiques d'activation a tendance à être de qualité relativement médiocre, et est ainsi peu valorisée sur le marché du travail. À l'opposé, certaines offres de formations peuvent aussi avoir un résultat positif, par exemple dans le cas de programmes sur mesure, conçus en lien étroit avec les besoins des employeurs locaux. Cependant, même dans ces conditions, le rendement salarial moyen de la formation délivrée dans le cadre de l'apprentissage en Allemagne, par exemple, n'est que de l'ordre de $10 \%$ par année de formation. Sauf cas exceptionnel, même un programme de qualité a peu de chances d'offrir un retour sur investissement sensiblement supérieur.

Du côté des points positifs, on peut supposer que les effets de déplacement entraînés par de tels programmes sont faibles. En effet, rien ne permet de penser qu'en offrant une formation à un groupe de personnes, on réduit les perspectives offertes aux autres. De fait, d'abondantes recherches suggèrent au contraire que les investissements dans le capital humain ont un effet d'entraînement positif (spillover effect) : plus les travailleurs qualifiés sont nombreux sur le marché, plus les salaires progressent pour l'ensemble des salariés.
En outre, les changements de comportement à long terme potentiellement occasionnés par la formation bénéficient en fin de compte aux actifs, y compris dans d'autres domaines que celui des revenus. Ainsi, il y a consensus sur le fait que la hausse du niveau de formation conduit à une amélioration de l'état de santé (Oreopoulos, 2007). Les formations proposées dans le cadre des politiques actives du marché du travail sont-elles de nature à entraîner de tels effets ? Les chercheurs ne se sont pas encore emparés de la question; nous supposons que l'on peut escompter des effets légers, mais positifs. Ce bénéfice est particulièrement vraisemblable en ce qui concerne les compétences de base, telles que l'alphabétisation, qui aident les salariés défavorisés à affronter un monde de plus en plus complexe.

\section{L’emploi direct, ou contrat aidé}

Qu'en est-il à présent des politiques de création directe d'emplois ? Ce terme général recouvre aussi bien les anciens dispositifs créant des emplois dits «occupationnels» que les stages assortis d'une indemnité subventionnée. Parmi les premières évaluations randomisées (2) des politiques d'activation, certaines ont été menées aux États-Unis afin de mesurer l'impact des dispositifs d'emploi direct visant les anciens délinquants et les jeunes très défavorisés (MDRC, 1980). Le raisonnement à l'origine de certains de ces dispositifs, dont justement ceux destinés aux ex-délinquants américains, est qu'une partie des individus concernés a besoin qu'on lui donne la chance de découvrir un environnement de travail classique afin de développer les habitudes de travail et les compétences sociales nécessaires à son insertion. D'autres dispositifs - parmi lesquels les plans d'embauche massive mis en place dans de nombreux pays au cours des années 1930 - ont d'abord trouvé leur justification en tant qu'outils de politique macroéconomique (on pense notamment à l'action de la Works Progress Administration sous la présidence de Roosevelt).

Quel type d'effets directs peut-on attendre de ces dispositifs ? Mis à part les meilleurs systèmes de stages, qui se rapprochent de formations en situation d'emploi, la probabilité d'un effet à long terme de ces mesures sur les participants est faible. Notons qu'elles peuvent même avoir tendance à leur nuire à long terme si l'on considère que certains participants, en l'absence d'un tel dispositif, auraient occupé durant le même temps des emplois standard, accumulant ainsi une expérience précieuse.

Les programmes d'emploi direct présentent également des risques élevés d'effets de

(2) $C f$. l'introduction du numéro pour une présentation plus détaillée des méthodes d'évaluation. 
déplacement, puisque les employeurs auraient offert les postes subventionnés à d'autres personnes si le programme n'avait pas été mis en place. Depuis les années 1970 et 1980, un certain nombre d'études ont pointé des effets de déplacement particulièrement importants pour le secteur public (CALMFORS, 1994). Bien souvent, ces programmes subventionnent des institutions publiques locales pour les inciter à placer sur certains postes des travailleurs aidés. Or, ces institutions peuvent avoir tendance à profiter des aides sans créer de nouveaux emplois, 1'effet de déplacement étant alors de $100 \%$.

\section{Bilan des trois types de politiques actives}

Le tableau 1 ci-dessous propose une synthèse des trois grands types de politiques actives du marché du travail, et fait apparaître leurs différences en termes de coûts pour les gouvernements, d'impact à court et à long terme et enfin, de risque d'effets de déplacement.

Tableau 1 : Politiques actives - Synthèse

\begin{tabular}{|l|c|c|c|}
\hline & $\begin{array}{c}\text { Accompagnement } \\
\text { à la recherche } \\
\text { d'emploi }\end{array}$ & Formation & $\begin{array}{c}\text { Emploi } \\
\text { direct }\end{array}$ \\
\hline Dépense publique & moyenne & $\begin{array}{c}\text { moyenne/ } \\
\text { élevée }\end{array}$ & élevée \\
\hline $\begin{array}{l}\text { Effet à court } \\
\text { terme }\end{array}$ & positif & négatif & positif \\
\hline $\begin{array}{l}\text { Effet à long } \\
\text { terme } \\
\text { (cas le plus } \\
\text { favorable) }\end{array}$ & faiblement positif & $\begin{array}{c}\text { positif } \\
(10 \%)\end{array}$ & $\begin{array}{c}\text { faible- } \\
\text { ment } \\
\text { positif }\end{array}$ \\
\hline $\begin{array}{l}\text { Effet à long terme } \\
\text { (cas le moins } \\
\text { favorable) }\end{array}$ & faiblement négatif & $\begin{array}{c}\text { faiblement } \\
\text { négatif }\end{array}$ & négatif \\
\hline Déplacement & moyen & faible & élevé \\
\hline
\end{tabular}

Du point de vue des dépenses publiques, les programmes d'aide à la recherche d'emploi sont très attractifs en raison de leur faible coût. Les programmes de formation ont un coût moyen à élevé tandis que les programmes d'emploi direct sont les plus coûteux.

À court terme, les dispositifs d'accompagnement à la recherche d'emploi paraissent avoir un effet bénéfique sur les revenus, tandis que les formations ont généralement un impact négatif à court terme, et les plans d'emploi direct un effet positif (à condition de prendre en compte l'emploi subventionné lui-même dans cet effet).

Concernant les effets à plus long terme, qui demeurent plus incertains, le tableau fait apparaître les résultats paraissant constituer les cas les plus et les moins favorables pour chacun des trois types d'intervention. À long terme, les dispositifs d'accompagnement peuvent avoir un effet faiblement positif dans le cas le plus favorable et un effet faiblement négatif dans le cas le moins favorable (où les participants se verraient par exemple contraints d'accepter des emplois sous-qualifiés, limitant ainsi leur future progression de carrière). Dans le cas le plus favorable, les formations pourraient avoir un effet positif relativement élevé à long terme (rendement de l'ordre de $10 \%$ ). Dans le plus mauvais cas, l'impact pourrait être négatif si la formation est de faible qualité. Enfin, dans le cas le plus favorable, les politiques d'emploi direct pourraient avoir un effet légèrement positif, notamment dans le cas des stages de bonne qualité. Dans le plus mauvais cas, elles pourraient avoir un effet négatif dû au confinement des individus ciblés dans des emplois publics sans perspective (dead end jobs).

Les effets de déplacement des programmes de formation sont susceptibles d'être relativement faibles, tandis que ceux des politiques d'emploi direct risquent d'être relativement élevés. Quant aux mesures d'accompagnement, elles se situent entre ces deux extrêmes, le phénomène s'accentuant en cas de marché du travail particulièrement atone.

Enfin, si le tableau ne prévoit pas de ligne spécifiquement consacrée au bien-être des participants, une comparaison entre les programmes sur ce point n'en reste pas moins intéressante. En moyenne, à court terme, les dispositifs d'accompagnement à la recherche d'emploi sont de façon générale probablement moins appréciés par les personnes concernées : on se souvient que la menace de «bénéficier» d'un tel programme suffit pour que certains demandeurs d'emploi retrouvent du travail. Dans certains cas pourtant, surtout lorsqu'ils ciblent les chômeurs de longue durée ou d'autres groupes défavorisés, ces dispositifs peuvent accroître la satisfaction des participants en leur donnant le coup de pouce nécessaire à une recherche plus efficace.

Les sessions de formation sont capables d'augmenter le sentiment de bien-être des participants, notamment lorsqu'ils n'ont pas à financer euxmêmes leur formation et lorsqu'ils peuvent en apprécier les retombées sous la forme d'un revenu plus élevé par la suite.

Enfin, les plans de création directe d'emplois améliorent la satisfaction des participants à court terme, sous réserve que le salaire perçu pour ce travail subventionné compense les coûts entraînés par la reprise du travail.

\section{Les enseignements généraux des recherches existantes}

Dans cette section, nous passons rapidement en revue l'abondante littérature consacrée à l'évaluation des politiques actives du marché du travail depuis la fin des années 1970, sans insister outre mesure sur la méthodologie de ces évaluations, 
ce travail ayant déjà été largement accompli (voir par exemple: HeckMan et al., 1999 ou l'introduction de ce numéro). Notons simplement qu'en règle générale, les dispositifs adoptés en Europe, directement liés aux systèmes d'assurancechômage ou aux autres revenus de substitution et le plus souvent obligatoires pour les participants, sont plus faciles à évaluer que les programmes habituellement mis en place aux États-Unis, à participation volontaire et ouverts à une grande variété de profils. La raison en est qu'en rendant la participation à ces dispositifs obligatoire, les pays européens permettent d'éliminer la plupart des biais présents dans les programmes fondés sur le volontariat.

Sur le fond, l'état de la recherche permet de tirer trois grandes conclusions (voir CARD et al., 2010 pour de plus amples détails, et Martin, GrubB, 2001; Greenberg et al., 2003, pour des analyses plus anciennes). Comme on pouvait s'y attendre a priori, les dispositifs d'aide à la recherche d'emploi ont en moyenne un effet positif à court terme, tandis qu'à long terme, leur effet est faible, voire nul. Les programmes d'emploi direct sont ceux dont les résultats sont les plus mauvais, à la fois à court terme (à condition d'exclure l'effet immédiat de l'emploi aidé lui-même) et à long terme. Enfin, concernant les offres de formation, les recherches suggèrent une action négative à court terme, mais positive à long terme.

Une réserve doit toutefois être émise concernant les effets à long terme: nombre d'études ne permettent pas d'évaluer des effets de 5 à $10 \%$ sur les revenus annuels. Ainsi, alors que beaucoup de programmes sont a priori de nature à entraîner un rendement de $10 \%$, la méthodologie des études et la taille trop réduite des échantillons empêchent de trancher sur ce point.

Le tableau 2 ci-après résume les effets estimés à court et à plus long terme des trois types de politiques, d'après la synthèse de David CARD, Jochen Kluve et Andrea Weber (2010) portant sur une centaine d'articles, correspondant à près de 200 études d'impact. Dans notre état de la recherche sur le sujet, mes coauteurs et moi-même avons mis au point une classification très simple en trois catégories, selon que le programme étudié obtient des effets significativement positifs, indéterminés, ou significativement négatifs.

L'analyse montre ainsi que $62 \%$ des dispositifs d'accompagnement à la recherche d'emploi ont un effet significativement positif à court terme (dans les douze mois suivant la fin du programme). En revanche, à court terme, formations et emplois aidés offrent un tableau particulièrement contrasté.
Tableau 2 : Synthèse des résultats des différents types de programmes (état de la recherche)

\begin{tabular}{|c|c|c|c|}
\hline & \multicolumn{3}{|c|}{$\begin{array}{c}\text { Pourcentage des programmes } \\
\text { à effet : }\end{array}$} \\
\hline & $\begin{array}{l}\text { significa- } \\
\text { tivement } \\
\text { positif (1) }\end{array}$ & $\begin{array}{l}\text { non signi- } \\
\text { ficatif (2) }\end{array}$ & $\begin{array}{c}\text { significa- } \\
\text { tivement } \\
\text { négatif (3) }\end{array}$ \\
\hline \multicolumn{4}{|c|}{ Impact à court terme estimé ( $\sim 12$ mois $)$} \\
\hline $\begin{array}{l}\text { a. Accompagnement à } \\
\text { la recherche d'emploi } \\
(\mathrm{N}=24)\end{array}$ & 62,5 & 29,2 & 8,3 \\
\hline $\begin{array}{l}\text { b. Programmes de } \\
\text { formation }(\mathrm{N}=77)\end{array}$ & 33,8 & 36,4 & 29,9 \\
\hline $\begin{array}{l}\text { c. Programmes d'em- } \\
\text { ploi direct }(\mathrm{N}=56)\end{array}$ & 33,9 & 26,8 & 39,3 \\
\hline \multicolumn{4}{|c|}{ Impact à moyen terme estimé ( 24 mois) } \\
\hline $\begin{array}{l}\text { a. Accompagnement à } \\
\text { la recherche d'emploi } \\
(\mathrm{N}=7)\end{array}$ & 57,1 & 42,9 & 0,0 \\
\hline $\begin{array}{l}\text { b. Programmes de } \\
\text { formation }(\mathrm{N}=57)\end{array}$ & 56,1 & 42,1 & 1,8 \\
\hline $\begin{array}{l}\text { c. Programmes d'em- } \\
\text { ploi direct }(\mathrm{N}=25)\end{array}$ & 40,0 & 28,0 & 32,0 \\
\hline
\end{tabular}

Source : CARD et al., 2010.

À moyen terme (soit environ deux ans après la fin du programme), les résultats des formations sont plus favorables. En effet, $56 \%$ de ces programmes trouvent une issue significativement positive. Les programmes d'emploi direct ne voient pas leurs résultats évoluer entre le court et le moyen terme, leur taux de résultats significativement positifs et négatifs étant d'ailleurs comparables. Finalement, ces programmes semblent en moyenne avoir peu d'impact, les effets positifs et négatifs sur les participants paraissant se compenser.

Tableau 3 : Relation entre impact à court et moyen terme des politiques actives du marché du travail offrant une formation

\begin{tabular}{|l|c|c|c|}
\hline & \multicolumn{3}{|c|}{ Pourcentage des effets à moyen } \\
terme : \\
\cline { 2 - 4 } & $\begin{array}{c}\text { significa- } \\
\text { tivement } \\
\text { positifs } \\
\text { (1) }\end{array}$ & $\begin{array}{c}\text { non signi- } \\
\text { ficatifs (2) }\end{array}$ & $\begin{array}{c}\text { significa- } \\
\text { tivement } \\
\text { négatifs } \\
\text { (3) }\end{array}$ \\
\hline Effet à court terme estimé & 86,8 & 13,3 & 0,0 \\
\hline $\begin{array}{l}\text { a. Significativement } \\
\text { positif (N =15) }\end{array}$ & 36,8 & 63,2 & 0,0 \\
\hline $\begin{array}{l}\text { b. Non significatif } \\
(\mathrm{N}=19)\end{array}$ & 42,1 & 52,6 & 5,3 \\
\hline $\begin{array}{l}\text { c. Significativement } \\
\text { négatif }(\mathrm{N}=19)\end{array}$ &
\end{tabular}

Remarque : L'échantillon contient des études où l'effet à court et moyen terme est estimé pour le même programme et le même groupe de participants

Source : CARD et al. (2010)

Le tableau 3 montre sous un autre angle la transition du court terme au plus long terme, en se focalisant uniquement sur les offres de formation. Ici, nous présentons un tableau croisé des effets à court terme d'un programme donné (sur les différentes lignes) et de ses résultats à moyen terme 
(proposés en colonnes). Parmi les programmes montrant un effet négatif significatif à court terme (ligne c), $42 \%$ ont un effet significativement positif à moyen terme, et seuls $5 \%$ un effet négatif à moyen terme (Нотz et al., 2006).

Cette synthèse suggère qu'un programme ayant un effet néfaste dans un premier temps peut ensuite avoir des effets bénéfiques. Pour illustrer cette transition, on évoquera par exemple le dispositif GAIN (Greater Avenues for Independence) destiné aux bénéficiaires des aides sociales en Californie. Des études correctement randomisées montrent que les personnes ayant bénéficié des formations dispensées dans ce cadre ont vu leurs revenus baisser à court terme, mais augmenter de façon substantielle à plus long terme.

\section{Enseignements récents provenant des États-Unis}

Cette section est consacrée à une rapide synthèse de quelques-uns des enseignements que peuvent nous apporter les recherches les plus récentes menées sur les politiques actives du marché du travail aux États-Unis.

\section{Formation et "choix individuel"}

Aux États-Unis, une forte tradition conduit à valoriser les choix individuels lorsqu'est proposée une grande variété d'offres. Une évaluation expérimentale de la valeur de ces choix a récemment été menée dans le cadre d'une analyse consacrée aux formations financées par le Workforce Investment Act (WIA) (Perez-Johnson et al., 2011). Le programme de base prévu par la loi propose à chaque participant un «compte formation» sur lequel est versée une somme destinée à financer différents programmes. Dans le cadre de cette expérimentation, différents groupes de participants se voyaient proposer différents niveaux d'intervention de la part des professionnels. Dans un premier groupe, les personnes étaient laissées libres de choisir le ou les plans de formation ayant leur préférence, en dépensant à leur guise la somme allouée. Pour d'autres participants, chacune des options qu'ils avaient choisies devait être validée par les agents chargés de leur suivi. Enfin, pour un troisième groupe, une solution intermédiaire était adoptée, nécessitant une forme d'accord entre les participants et leur conseiller, avec une marge de négociation possible. Le principal enseignement de cette évaluation a été qu'accroître la supervision du choix par des professionnels a pour effet d'augmenter de façon significative les revenus des participants.

Ce résultat semble confirmer l'opinion partagée par les économistes comportementalistes, selon lesquels le choix individuel non restreint n'est pas forcément la meilleure option, surtout dans le cas de choix relativement complexes où l'individu décisionnaire ne possède qu'une expérience limitée des différentes options en présence et de leurs conséquences possibles.

\section{Créer son propre emploi : l'entreprise individuelle}

La création d'une entreprise individuelle est souvent évoquée comme une solution possible aux difficultés rencontrées par les travailleurs déclassés. Le programme expérimental GATE (Growing America Through Entrepreneurship) a récemment été conçu dans le but de tester les résultats de mesures encourageant les chômeurs à monter leur propre entreprise (BENUs et al., 2008). Le bilan de cette évaluation est plutôt négatif : la politique d'incitation a certes conduit à une augmentation du nombre de créations d'entreprises, mais aussi à une baisse des revenus et à une hausse des dépenses de l'État.

\section{Les jeunes défavorisés : un cas difficile}

Un troisième enseignement des recherches récentes conduites aux États-Unis est que concevoir une politique véritablement efficace pour aider les jeunes défavorisés est un exercice particulièrement difficile. Nombre de pays, dont la France, sont confrontés à un taux de chômage élevé chez les jeunes, a fortiori chez les jeunes migrants ou appartenant aux minorités. Dans le contexte de la «guerre contre la pauvreté» ("War on Poverty») menée dans les années 1960, une mesure phare a été la création des "Job corps », un programme intensif de formation visant les jeunes de 16 à 24 ans faiblement qualifiés et jouissant de faibles revenus. En règle générale, les participants ont bénéficié du programme pendant un an, pour un coût moyen de 20000 dollars.

Or, au milieu des années 1990, une évaluation randomisée à grande échelle menée sur les $J o b$ corps (SCHOCHET et al., 2008) a conclu à l'efficacité du programme à court terme, mais aussi à la dilution de ses effets quelques années à peine après la fin de l'intervention. Les raisons précises de ce phénomène sont encore débattues.

\section{Lorsque les réformes passives fonctionnent}

Un quatrième enseignement qui nous semble découler de la littérature récente est qu'une réforme du système «passif» de revenus peut constituer une façon peu coûteuse d'encourager le travail et de réduire le recours aux programmes actifs. En particulier, les éléments disponibles sur les programmes d'incitation financière comme le Earned Income Tax Credit (3) ainsi que les initiatives ciblées, comme

(3) L'Earned Income Tax Credit est un crédit d'impôt octroyé aux foyers ayant des revenus du travail modestes et, pour la plupart d'entre eux, des enfants à charge. 
celles permettant de cumuler salaires et prestations sociales (enhanced earnings disregards) semblent avoir un impact important sur les comportements par rapport au travail (BLANK et al., 2000).

Des évaluations randomisées de toute une série d'incitations financières (dont une incitation à l'emploi s'adressant aux bénéficiaires des aides sociales à long terme au Canada), suggèrent que les incitations financières contribuent à augmenter à la fois le taux d'emploi, le niveau de revenu et le bienêtre des participants. Reste ce qui peut apparaître comme un inconvénient de ces politiques, à savoir leur coût important. Aussi les initiatives de ce type sont-elles moins attractives dans un contexte fiscalement tendu.

Le programme canadien fait également apparaître un élément intéressant : la combinaison d'une incitation financière et d'une mesure active (accompagnement à la recherche d'emploi) peut s'avérer extrêmement efficace. C'est donc dans ce domaine qu'il conviendrait d'intensifier tout particulièrement l'expérimentation et la recherche.

\section{Et maintenant ? Les innovations en Europe}

Au cours de la prochaine décennie, la recherche sur les politiques actives du marché du travail devrait se concentrer fortement sur l'Europe et ce, pour plusieurs raisons. D'une part, l'ère des idées entièrement nouvelles et de l'expérimentation sociale apparaît largement révolue aux États-Unis. D'autre part, la persistance d'un chômage de masse dans bon nombre de pays européens a déjà eu pour effet d'encourager grandement l'expérimentation, processus qui devrait se poursuivre dans les années à venir.
Trois nouvelles questions semblent se dessiner, que des travaux récents particulièrement stimulants ont déjà en partie abordées.

Premièrement, qui, du secteur public ou privé (ou des deux), devrait être chargé des politiques actives du marché du travail ? Ce débat déjà ancien a toujours été particulièrement vif aux États-Unis. On ne peut que se réjouir des apports de nouvelles expérimentations conduites en France, afin de mieux appréhender la nature et l'origine des différences entre acteurs publics et privés sur ce terrain (Behaghel et al., 2012).

Deuxièmement, quelle est l'ampleur des effets de déplacement? Encore une fois, il s'agit là d'un problème ancien récemment revenu sur le devant de la scène grâce à une expérience novatrice menée en France (CRÉPON et al., 2013). Ce sujet fera, sans nul doute, l'objet d'une attention croissante au cours des prochaines années.

Troisièmement, quel est le rôle joué par les travailleurs sociaux ? Le champ de l'économie comportementale a fourni, selon nous, une base solide pour la compréhension des difficultés rencontrées par les individus opérant des choix dans un environnement complexe. On peut soutenir que dans de tels contextes, les travailleurs sociaux ont un véritable rôle à jouer en aidant les personnes concernées à s'informer et à prendre des décisions en toute connaissance de cause. Ils ont aussi l'opportunité de les aider à dépasser un certain nombre de biais comportementaux (procrastination, tendance à privilégier le présent, etc.), que ce soit par des «coups de pouce» décisifs ( "nudge») ou par d'autres types d'interventions. Les quelques années qui s'annoncent devraient ainsi voir fleurir d'intéressantes recherches portant sur la manière dont les professionnels accomplissent leur travail et sur la mission qui leur revient dans l'amélioration de l'efficacité des divers programmes, y compris ceux relevant des politiques actives du marché de l'emploi.

\section{Bibliographie}

BECKER G. S. (1962), «Investment in human capital: A theoretical analysis», Journal of political economy, vol. 70, $\mathrm{n}^{\circ} 5$, part 2, pp. 9-49.

Behaghel L., Crépon B., Gurgand M. (2012), «Private and public provision of counseling to job-seekers: evidence from a large controlled experiment», IZA Discussion paper, $\mathrm{n}^{\circ} 6518$.

Benus J., McConnell S., Bellotti J., Shen T., Fortson K., KahVecioglu D. (2008), « Growing America through entrepreneurship: findings from the evaluation of project GATE», Unpublished report, Mathematica Policy Research.
Black D. A., Smith J. A., Berger M. C., Noel B. J. (2003), «Is the threat of reemployment services more effective than the services themselves? Evidence from random assignment in the UI system», American economic review, vol. 93, n 4, pp. 1313-1327.

Blank R. M., CARD D., Robins P. K. (2000), «Financial incentives for increasing work and income among low-income families », in Blank R. M., Card D. (ed.), Finding work: jobs and welfare reform, New York, Russell Sage Foundation, pp. 373-419. 
CAlmfors L. (1994), «Active labour market policy and unemployment: a framework for the analysis of crucial features », OECD economic studies, $\mathrm{n}^{\circ} 22$, pp. 7-47.

CARD D. (1999), «The causal effect of education on earnings », in Ashenfelter O. C., Card D. (ed.), Handbook of labor economics, vol. 3, Part A., Amsterdam, Elsevier, pp. 1801-1863.

Card D., Kluve J., Weber A. (2010), «Active labour market policy evaluations: a meta-analysis », The Economic journal, vol. 120, n 548 , pp. F452-F477.

Crépon B., Duflo E., Gurgand G., Rathelot R., Zamora P. (2013), «Do labor market policies have displacement effects? Evidence from a clustered randomized experiment», Quarterly journal of economics, vol. 128, $\mathrm{n}^{\circ} 2$, pp. 531-580.

Greenberg D., Ashworth K., Cebulla A., Walker R. (2005), «When welfare-to-work programs seem to work well: explaining why riverside and portland shine so brightly», Industrial and labor relations review, vol. 59, $\mathrm{n}^{\circ} 1$, pp. 34-53.

Greenberg D., Michaloupoulos C., Robins P. K. (2003), «A meta-analysis of government-sponsored training programs», Industrial and labor relations review, vol. $57, \mathrm{n}^{\circ} 1$, pp. 31-53.

Heckman J. J., Lalonde R. J., Smith J. A. (1999), «The economics and econometrics of active labor market programs », in Ashenfelter O. C., Card D. (ed.), Handbook of labor economics, vol. 3, Part A., Amsterdam, Elsevier, pp. 1865-2097.

Hotz V. J., Imbens G., KleRman J. A. (2006), «The long term gains from GAIN: a re-analysis of the impacts of the California GAIN program», Journal of Labor Economics, vol. 24, n 3 , pp. 521-566.

KLuve J. (2010), «The effectiveness of European active labor market programs», Labour economics, vol. 17, $\mathrm{n}^{\circ}$ 6, pp. 904-918.
Krueger A. B., Mueller A. (2011), « Job search and job finding in a period of mass unemployment: evidence from high-frequency longitudinal data», CEPS Working Paper, $\mathrm{n}^{\circ} 215$.

LALONDE R. J. (1995), «The promise of public sectorsponsored training programs », Journal of economic perspectives, vol. 9, $\mathrm{n}^{\circ}$ 2, pp. 149-168.

LeChNer M., Wunsch C. (2009), «Are training programs more effective when unemployment is high?», Journal of labor economics, vol. 27, n 4, pp. 653-692.

Manpower Demonstration Research Corporation (MDRC) (1980), Summary and findings of the National Supported Work Demonstration, Cambridge MA, Ballinger.

Martin J. P., GrubB D. (2001), «What works and for whom? A review of OECD countries' experiences with active labour market policies », Swedish economic policy review, vol. $8, \mathrm{n}^{\circ} 2$, pp. 9-56.

MinCER J. (1958), «Investment in human capital», Journal of political economy, vol. 66, n 4, pp. 281-302.

Oreopoulos P. (2007), «Do dropouts drop out too soon? Wealth, health and happiness from compulsory schooling», Journal of public economics, vol. 91, $\mathrm{n}^{\circ}$ 11-12, pp. 2213-2229.

Perez-Johnson I., Moore Q., Santillano R. (2011), «Improving the effectiveness of individual training accounts: long-term findings from an experimental evaluation of three service delivery models», Final report, Princeton NJ, Mathematica Policy Research, October.

Schochet P. Z., Burghardt J., McConnell S. (2008), «Does the Job Corps work? Impact findings from the National Job Corps study», American economic review, vol. $98, \mathrm{n}^{\circ} 5$, pp. 1864-1886. 